\title{
THE EFFECTS OF FILTERING ON HIGH FREQUENCY OSCILLATION CLASSIFICATION
}

\author{
Jiaju Liu \\ Rachael Garner BA \\ Marianna La Rocca PhD \\ Eun-Kee Bae MD PhD \\ Dominique Duncan PhD
}

Laboratory of Neuro Imaging

USC Stevens Neuroimaging and Informatics Institute

Keck School of Medicine of USC

University of Southern California

2025 Zonal Avenue, Los Angeles, CA, USA

jiajuliu1@gmail.com; \{rgarner, mlarocca, eun-kee.bae, dduncan\}@loni.usc.edu

\begin{abstract}
High frequency oscillations (HFOs) have been used for seizure prediction and are promising biomarkers of epileptogenesis. However, detecting HFOs is time consuming and subjective, prompting research into automated detection and classification pipelines. We aim to understand how different EEG filtering methods impact these pipelines and harmonize detections from the same data when preprocessed differently. We preprocessed EEG with two different filters and then detected events with the short time energy (STE) detector and compared common detections. We applied t-distributed stochastic neighbor embedding (t-SNE) to the datasets and compared embeddings then investigated if shifting commonly detected events prior to t-SNE helped standardize embeddings. The finite impulse response (FIR) and infinite impulse response (IIR) filters achieved a Cohen's Kappa coefficient of 0.8962 after shifting, reflecting a high level of agreement. The t-SNE embeddings were similar only when data were shifted prior to embedding. Feasible solutions to this shifting problem are addressed.
\end{abstract}

Keywords: high frequency oscillations, EEG, epilepsy, finite impulse response filter, infinite impulse response.

\section{INTRODUCTION}

Traumatic brain injury (TBI) patients can experience secondary pathologies, including posttraumatic epilepsy (PTE), or unprovoked seizures following a TBI (Garner et al. 2019). Pathological high frequency oscillations (HFOs) found in electroencephalography (EEG) recordings have been used to detect regions of epileptogenesis or seizure onset zones ( $\mathrm{Li}$ et al. 2018; Liu et al. 2018; Gliske et al. 2016; Worrel et al. 2004; Cimbálník et al. 2018; Birjandtalab et al. 2016; Liu et al. 2015) and are promising potential biomarkers of epileptogenesis (Vespa et al. 2013; Agrawal et al. 2006). These waveforms are transient bursts of activity between $80-500 \mathrm{~Hz}$ that can be distinguished from background EEG (Frauscher et al. 2017). However, detecting HFOs is exceedingly difficult. Visual detection of HFOs is time consuming-requiring up to 10 hours to visually analyze a 10-minute 10 channel recording (Zelmann et al. 2012). Even experienced epileptologists have biases that cause poor interrater agreement (Spring et al. 2017). The subjective, ill- 
defined nature of choosing when a segment of data "stands out" (Frauscher et al. 2017) questions the strength of studies that depend on the validity of their annotations. As a result, visual analyses are not feasible for large datasets, such as the Epilepsy Bioinformatics Study for Antiepileptogenic Therapy (EpiBioS4Rx) (Duncan et al. 2019), which collects continuous EEG for 7-14 days in human TBI patients and $30+$ days in a preclinical rodent model.

Recent studies have addressed the burden of manual HFO detection by implementing automatic HFO detectors, which identify precise, numerical traits associated with HFOs (Zelmann et al. 2012; Dirodi et al. 2019; Worrel et al. 2008). Automated HFO detectors, such as RippleLab (Navarrete et al. 2016), a widely used detector, are imperfect due to the incredibly diverse range of filtering and processing methods that affect detected waveforms. One inconsistency lies in bandpass filter frequency limits: studies set bandpass filter limits from $80 \mathrm{~Hz}-160 \mathrm{~Hz}$ (Dirodi et al. 2019) to $80 \mathrm{~Hz}-1000 \mathrm{~Hz}$ (Worrel et al. 2008) and many in between. Different studies also use various types of bandpass filters, namely the finite impulse response (FIR) filters (Staba et al. 2002, Zelmann et al. 2011, Crepon et at. 2010, Jacobs et al. 2008) and infinite impulse response (IIR) filters (Worrel et al. 2008). Varying the filtering methods applied to raw EEG affects the waveforms supplied to automated HFO detectors.

Developing a standardized HFO preprocessing and detection pipeline will allow HFO detectors to be compared across laboratories, ultimately resulting in a more quantitatively rigorous and comprehensive definition of HFOs. While Zelmann et al. 2011 compared HFO detectors to find an optimal detector, our work focuses on standardizing HFO detection methods. The goal of this study is to discover the effects of filtering methods on automated HFO detection. In addition, this study aims to minimize the variance introduced by different filters and harmonize detections produced from the same data that have been preprocessed in different ways.

\section{METHODS}

\subsection{Data}

EEG data were collected from a patient who had suffered a moderate-severe traumatic brain injury (TBI) and later developed posttraumatic epilepsy (PTE). The data were sampled at $2000 \mathrm{~Hz}$, with a duration of 6 hours and 5 minutes, and were collected from the University of California, Los Angeles for the Epilepsy Bioinformatics Study for Antiepileptogenic Therapy (EpiBioS4Rx) (Duncan et al. 2019).

\subsection{Data Preprocessing}

Two datasets were formed from the original data: one was created by applying MATLAB's EEGLAB (Delorme et al. 2004) FIR filter, and the other was created by applying MATLAB RippleLab's (Navarrete et al. 2016) IIR filter. Both toolboxes use bandpass filters between $80 \mathrm{~Hz}$ and $500 \mathrm{~Hz}$ and do not introduce phase delay. The next two steps, HFO detection and t-distributed stochastic neighbor embedding (t-SNE), are performed on each dataset.

\subsection{HFO Detection}

HFOs were identified using a highly sensitive but nonspecific energy-based detector proposed by Staba et al. (Staba et al. 2002). Implemented in RippleLab (Navarrete et al. 2016), the detector divides data from each channel into 180-second epochs. Within each epoch, root-mean-square (RMS) energy values are computed for successive $3 \mathrm{~ms}$ nonoverlapping windows. Segments are identified as HFOs if they 1) maintain energy greater than 5 standard deviations above mean RMS, 2) longer than $6 \mathrm{~ms}$, and 3) contain more than 8 combined peaks and troughs. Events occurring less than $10 \mathrm{~ms}$ apart were combined to form 
one event. Finally, segments were standardized to span $150 \mathrm{~ms}$ before and after the midpoint of the original detection to form the data matrix for subsequent unsupervised clustering.

\section{4 t-Distributed Stochastic Neighbor Embedding}

Detecting relevant HFOs is exceedingly difficult. Physiological HFOs are involved in the common tasks of memory formation and information processing (Zijlmans et al. 2012). Moreover, high-frequency bursts are also exhibited following peripheral stimulation (Baker et al. 2003). Finally, artifacts and non-HFO waveforms are often erroneously detected by modern HFO detectors. As a result, some studies have applied dimensionality reduction methods to discover patterns in the data that distinguish these categories (Liu et al. 2018). This provides insight into the entire dataset and saves time by automatically separating artifacts, non-HFO detections, and sorting HFOs into groups.

One approach that has become popular for visualizing high dimensional data is the t-distributed stochastic neighbor embedding (t-SNE) algorithm (Van Der Maaten et al. 2015). t-SNE defines a pairwise similarity measure between points and finds a low-dimensional embedding that roughly preserves this metric on a local scale. The goal of t-SNE is to minimize the difference between the original dataset and lowdimensional embedding. As t-SNE has been a powerful tool in classifying images and speech but has not yet been applied to HFO classification, we apply it to the waveforms detected by the HFO detector to see if it is affected by preprocessing methods. With t-SNE as a model for other machine learning algorithms, understanding how discepancies in HFO detectors and filtering methods affect subsequent machine learning will provide insight into the growing field of automated HFO classification, a much faster, cheaper alternative to visual detection.

Following HFO detection on the 6 hour and 22 minute EEG file, the $\mathrm{n}$ detected waveforms each of length $\mathrm{m}=300(2000 \mathrm{~Hz} * 0.15 \mathrm{sec})$ form an $m \times n$ data matrix $\mathrm{X}=\left\{x_{1}, x_{2}, \ldots, x_{n}\right\}$. The kernel applied in t-SNE is computed as follows. First, the nonsymmetric Gaussian kernel

$$
K_{i j}=k\left(x_{i}, x_{j}\right)=\exp \left(-\left\|x_{i}-x_{j}\right\|_{2}^{2} / 2 \sigma_{i}^{2}\right)
$$

is reintroduced with a different parameter $\sigma_{i}$ that will be explained later. From here, a normalized Markov matrix $P_{i j}=p(i, j)$ is constructed on the dataset and can be interpreted as the probability that observation $x_{i}$ will diffuse to $x_{j}$ in a random walk. We set

$$
p(i, j)=\frac{k(i, j)}{\sum_{l \neq i} k(i, l)},
$$

with $p(i, i)=0$. Then the $m \times m$ symmetric kernel $W=[w(i, j)]$ employed in t-SNE is defined as

$$
w(i, j)=\frac{p(i, j)+p(j, i)}{2 n} .
$$

Returning to the kernel parameters, each $\sigma_{i}$ is chosen such that the perplexity of the $i^{\text {th }}$ column of $W$ is equal to some predetermined value $u$.

Datasets can be reduced to a smaller three dimensions, for example, and visualized in such a way that relative distances are preserved. However, this is not always true for data sampled from an intrinsically high-dimensional (more than three dimensions) dataset since the region of all points equidistant from a center grows with distance much faster in high dimensions than in low dimensions. 
This is defined as the "crowding problem" (Van Der Maaten et al. 2008) and addressed by defining similarity in the low-dimensional space with a heavy-tailed distribution instead of a Gaussian. We let the low-dimensional complements to the original dataset $X=\left\{x_{1}, x_{2}, \ldots, x_{n}\right\}$ be $Y=\left\{y_{1}, y_{2}, \ldots, y_{n}\right\}$ respectively. The similarity $s(i, j)$ between $y_{1}$ and $y_{2}$ is defined in terms of the student-t distribution with one degree of freedom:

$$
s(i, j)=\frac{1}{\pi\left(1+\left\|y_{i}-y_{j}\right\|^{2}\right)}
$$

After normalizing (4) to form the low-dimensional kernel $Q_{i j}=q(i, j)$ where

$$
q(i, j)=\frac{s(i, j)}{\sum_{k \neq l} s(i, l)}, \quad q(i, i)=0
$$

t-SNE attempts to choose $\left\{y_{1}, y_{2}, \ldots, y_{n}\right\}$ such that the joint distributions $Q$ and $P$ are as similar as possible. This is quantified by minimizing their Kullback-Liebler divergence

$$
C(Y)=K L(P \| Q)=\sum_{i=1}^{n} \sum_{j=1, j \neq i}^{n} p(i, j) \log \frac{p(i, j)}{q(i, j)}
$$

using a Barnes-Hut algorithm.

\subsection{Comparison of Filtering Methods}

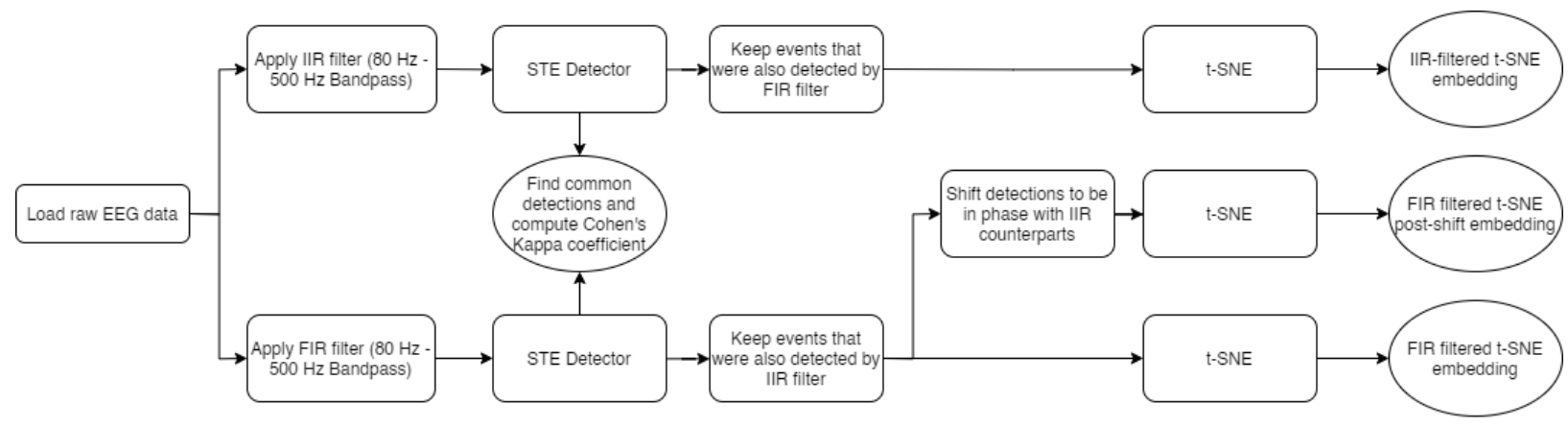

Figure 1: A visual of the HFO detection and comparison pipeline.

To understand the influence of filtering methods on the STE detector, we filtered raw EEG with two different filters (IIR and FIR) to create two preprocessed EEG files. We detected events with the STE detector, then identified and compared common detections. We measured the degree of agreement between the two filters with Cohen's Kappa coefficient, $\kappa$. This statistic ranges from 0 (agreement purely by chance) to 1 (perfect agreement) and is more effective at measuring the percentage agreement between two detectors because it adjusts for agreement due to chance (McHugh 2012).

To understand the influence of filtering methods on subsequent unsupervised classification, we analyzed and compared the t-SNE maps constructed from HFO detections obtained after the application of the two different filters. We examined similarities in terms of phase, cluster organization and number of detected events and waveforms. In addition, we evaluated if shifting the FIR-filtered detections to make the two 
filters in phase improves the similarity level. A MATLAB script was written to first automatically shift each FIR-filtered waveform to match its corresponding IIR-detection's phase, then compute the t-SNE embedding of the shifted FIR-filtered dataset. Indicated in the visual pipeline, three t-SNE embeddings will be computed.

\section{RESULTS}

\subsection{FIR vs. IIR Filtering Effects}

5,730 events were detected by the FIR filter, and 5,789 events were detected by the IIR filter. 2,428 waveforms were detected by both filters. The resulting Cohen's Kappa coefficient was $\kappa=0.4185$, reflecting a "minimal" to "weak" level of agreement far below the minimum value of $\kappa>0.8$ (Mchugh 2012).

Shifting the detections markedly improved interdetector agreement. When we extended our classification of similar detections to include all waveforms pairs detected within $10 \mathrm{~ms}$ of each other-the STE detector's minimum distance needed to separate events - the number of commonly detected pairs rose to 5,157 . The new Cohen's Kappa coefficient was $\kappa=0.8962$, bordering "almost perfect" agreement and far above $\kappa=0.8$ and the average human reviewer agreement of $\kappa=0.403$ (Spring et al. 2017). The mean time displacement of the 2,729 newly included $(52.92 \%$ of the new dataset) pairs was $1.135 \pm 1.706 \mathrm{~ms}$. Figure 1 compares one waveform as it was detected by the two filtering methods. After realignment, there is almost no difference between the two versions of the waveform. Also, the Euclidean distance used in the t-SNE algorithm was found to be highly dependent on small shifts in phase.

Comparison of FIR and IIR Filtered Detections
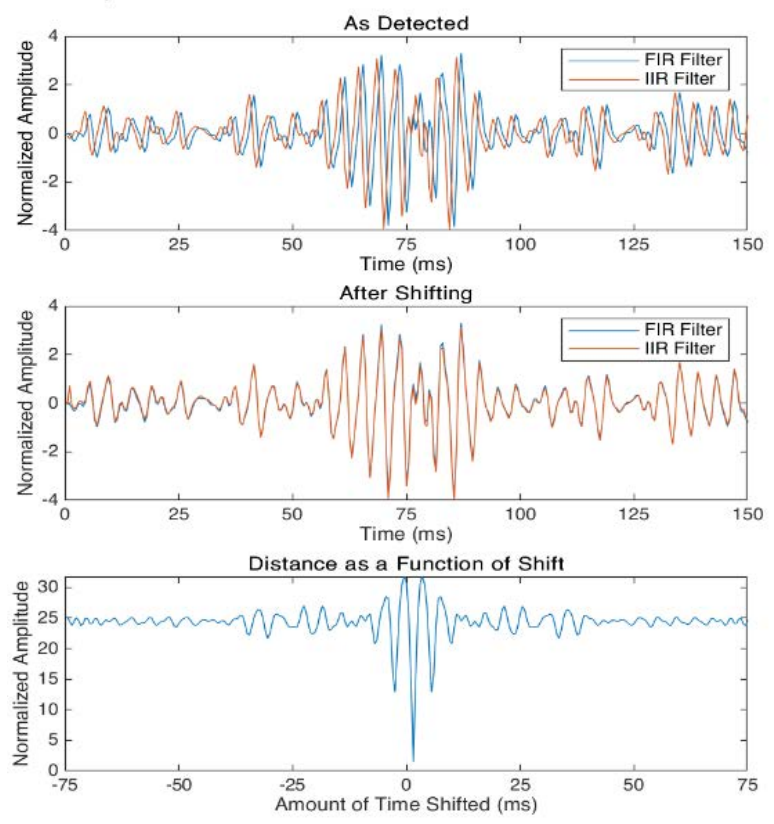

Figure 2: Feature vectors from FIR and IIR filtered detections plotted in the same window as they were detected (top) and after realignment (middle). (Bottom) the Euclidean distance between detections as a function of how far they are shifted. Since the detections are almost identical, their distance should be close to zero, which is reached by shifting $1.5 \mathrm{~ms}$ to be in phase. 


\subsection{Understanding How to Compare t-SNE on the Same Input Data}

One major implication of minimizing the Kullback-Liebler divergence, a nonconvex objective function, is that t-SNE will produce different results when run on the same dataset. However, t-SNE remains able to preserve the local geometry between similar detections, keeping its ability to separate data into groups very consistent. These facts can be seen when t-SNE is run twice on the dataset composed of IIR-filtered detections in Figure 3. On the left, clusters are formed, and each is a assigned a different color. t-SNE is then run again on the same dataset t-SNE is applied to the same dataset, but the resultant embeddings differ. However, overall geometry differs, every cluster that can be identified in the first trial appears in the second trial.

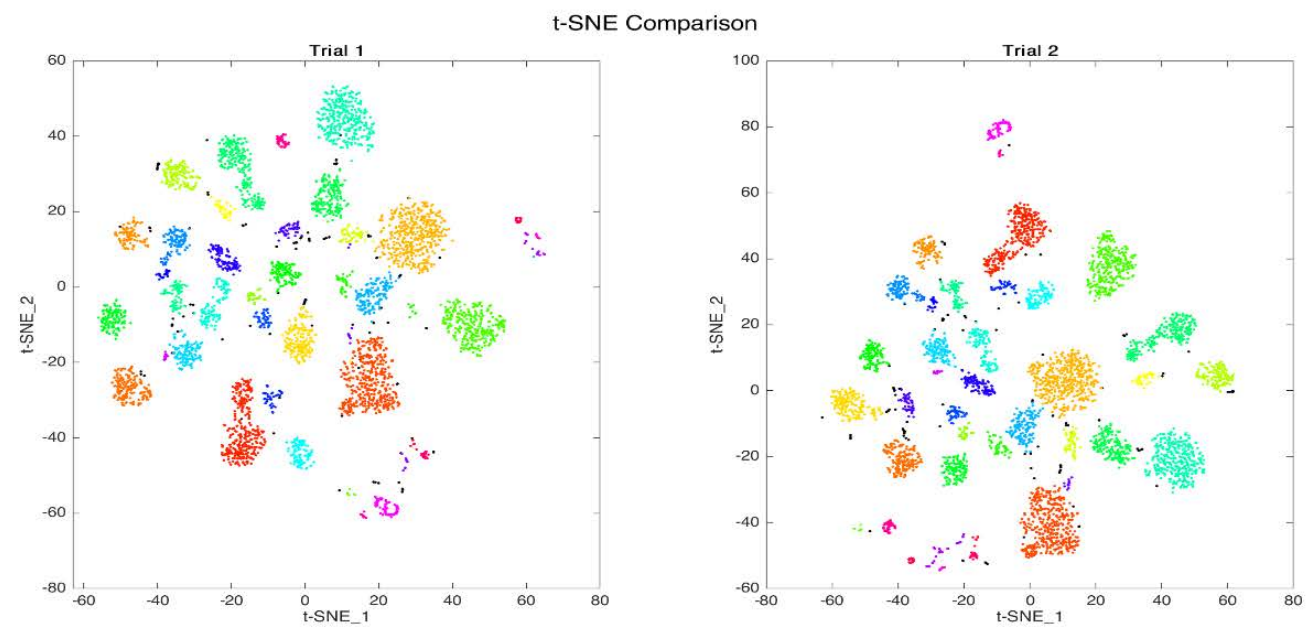

Figure 3: t-SNE embeddings when run on IIR-filtered detections twice. In trial 1 (left), clusters are identified and intracluster detections are assigned the same color. In trial 2 (right), t-SNE is run again and the detections retain the color they were previously assigned. Note that the axes, t-SNE_1 and t-SNE_2, do not have individual interpretations. Rather, t-SNE focuses on the relative location of points, embedding similar detections to the same cluster and embedding different detections relatively far away. 


\subsection{Comparing t-SNE on Differently Filtered Input Data}

Figure 3 shows the similarities between the embeddings when computed after applying different types of preprocessing.
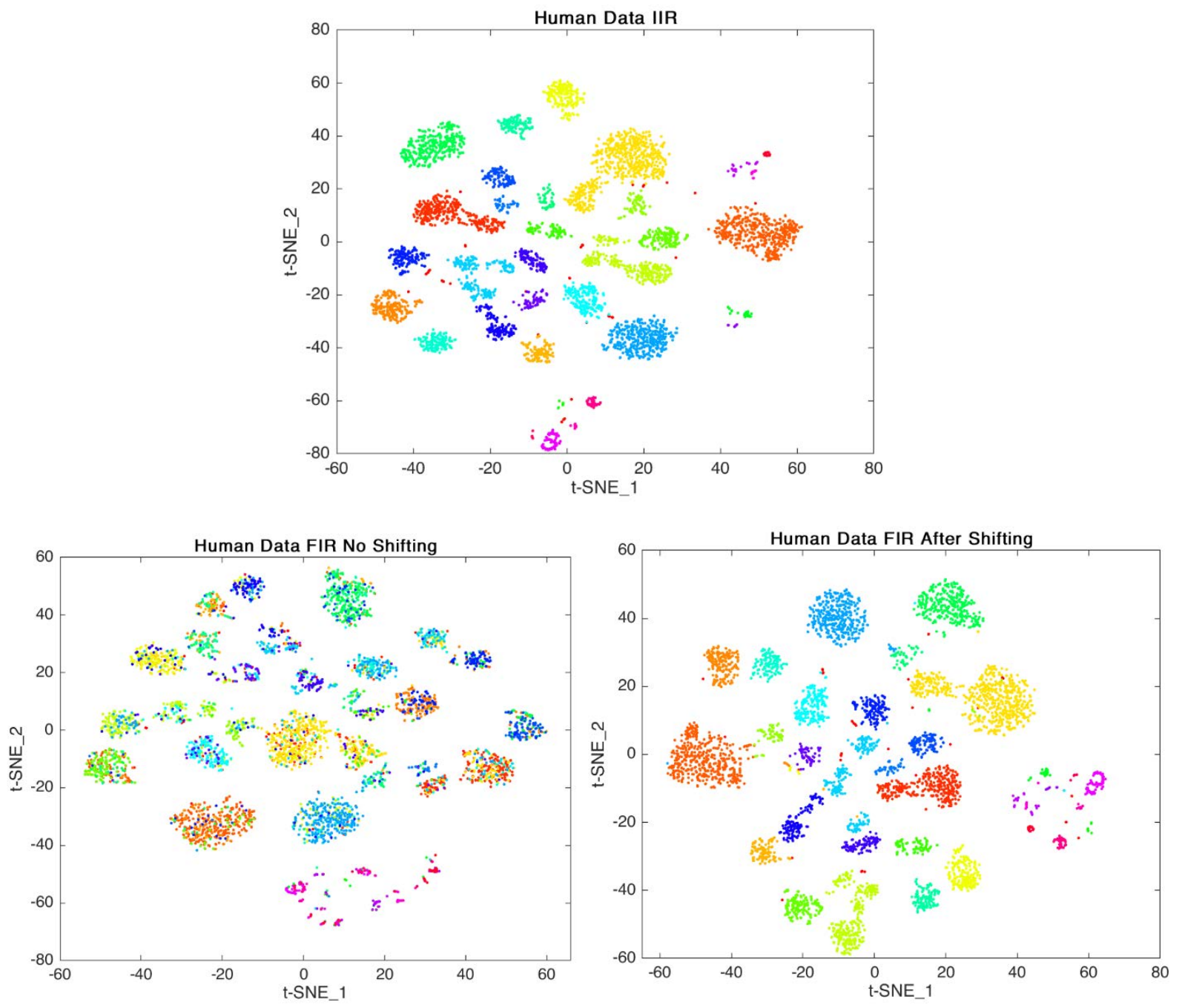

Figure 4: (Top) t-SNE embedding when applied to IIR-filtered detections. The detections are associated with the naturally occurring cluster that they appear in and colored accordingly. (Bottom right) t-SNE embedding when applied to FIR-filtered detections. The detections have the same color as their corresponding detections in the top t-SNE plot. (Bottom left) t-SNE embedding when applied to FIRfiltered detections that are shifted to be in phase with their corresponding IIR-filtered detections prior to running t-SNE. The detections have the same color as their corresponding detections in the top t-SNE plot.

Each embedding was computed from the 5,157 detections in its category that were detected throughout the 6 hour and 5 minute file. Based on the datasets used in van der Maaten et al. 2008 that range from 400 to 6,000 observations, we can be sure that enough data was inputted into the t-SNE algorithm. Although their common waveform morphologies were almost identical, the IIR-filtered (top) and FIR-filtered (left) t-SNE embeddings show drastically different results with little correspondence on global or local scales. In contrast, the IIR-filtered (top) and shifted FIR-filtered (right) t-SNE embeddings produced highly similar results. While the global structure of the embeddings is different, every cluster that can be identified in the embedding on the top has a corresponding location in the embedding on the right. In addition, uniformly 
colored clusters in both embeddings show that points embedded to the same cluster in one embedding are also embedded to the same cluster in the other embedding. Even though two different types of filters were used, their post-shifting t-SNE embeddings were very similar.

\section{DISCUSSION AND CONCLUSIONS}

This study contains three main contributions: identification of a problem, examples of its clinical relevance, and proposed solutions.

\subsection{The Effects of Filtering on HFO Classification}

This study's preliminary results point out the prominence of relative phase on automated HFO classification. Two commonly used filters, RippleLab's IIR filter and EEGLAB's FIR filter, as preprocessing tools for the STE detector were studied. The waveforms detected using each method showed a high level of agreement. Further examination of corresponding waveforms from each method revealed almost identical post-filtering morphology and differed most noticeably by their respective phases, yet their corresponding t-SNE embeddings clustered different groups of HFOs together with little agreement between the embeddings. As shifting the FIR-filtered data to match the phases of their IIR-filtered counterparts resolved this issue, we can conclude that phase, not the changes in morphology due to filtering, are responsible for the clustering discrepancy. Thus, we can conclude that IIR and FIR filters can be equally reliable if the problem of phase discrepancies is resolved.

\subsection{Relevance}

The problem of phase discrepancies is highly relevant to machine learning (ML) research aimed at classifying HFOs. Recently, researchers have looked to ML methods to automatically classify these detections (Pearce et al. 2013, Zuo et al. 2019), drastically reducing the time needed to find HFOs and removing human bias. However, our results show that the Euclidean distance is extremely sensitive to phase when comparing rapidly oscillating data. This is important since the Euclidean distance function is used in many machine learning methods: k-means, k-medoids, principal component analysis, t-SNE, Sammon maps, diffusion maps, isomaps, and support vector machines. The relatively new introduction of ML methods to automatic HFO classification combined with the fast-oscillatory behavior unique to HFOs may explain why this concern has not previously been studied. By demonstrating that t-SNE, a popular machine learning algorithm, is highly susceptible phase discrepancies to when applied to HFOs, the preliminary results of this study elucidate a major standardization problem that should be considered when constructing new HFO classification tools.

Additionally, our study's simple design, following a standard preprocessing, detection, and classification pipeline, allows its results to apply to all HFO classification studies. This study also analyzed clinical data, and thus its concerns regarding phase discrepancies are more easily translated to clinical studies than basic science studies, which are conducted on relatively clean rodent EEG.

\section{SOLUTIONS AND FUTURE WORK}

One option for standardizing HFO classification pipelines is to standardize every step of the HFO detection pipeline from data collection to machine learning. However, this is infeasible due to the large number of different HFO researchers. Standardized required equipment will limit application in many hospitals, especially hospitals in less wealthy neighborhoods that cannot afford new, likely high-end equipment. Finally, the proposed method will remain heavily influenced by the relative phase of detections within the dataset. 
Another solution is for future HFO classifiers to use ML algorithms that are not heavily affected by the detection's phase. Although this may also seem infeasible, convolutional neural networks are mostly shiftinvariant and have seen even more overall success at classifying data than t-SNE. In addition, ML algorithms that use a Euclidean distance metric may incorporate a cross-correlation-based metric that is equivalent to the Euclidean distance when detections are identical. Based on the preliminary results of shifting data and applying the t-SNE algorithm, both options are found to be promising and warrant further investigation.

\section{ACKNOWLEDGMENTS}

This study was conducted with the support of the National Institute of Neurological Disorders and Stroke (NINDS) of the National Institutes of Health (NIH) under award numbers R01NS111744 and U54 NS100064 (EpiBioS4Rx).

\section{REFERENCES}

Agrawal, A., J. Timothy, L. Pandit, and M. Manju. 2006. "Post-traumatic epilepsy: An overview". Clinical Neurology and Neurosurgery, vol. 108, pp. 433-439.

Baker, S. N., C. Gabriel, and R. N. Lemon. 2003. "EEG oscillations at $600 \mathrm{~Hz}$ are macroscopic markers for cortical spike bursts". The Journal of Physiology, vol. 550, pp. 529-534.

Birjandtalab, J., M. B. Pouyan, and M. Nourani. 2016. "Nonlinear dimension reduction for EEG-based epileptic seizure detection". In Proceedings of the 2016 IEEE-EMBS International Conference on Biomedical and Health Informatics (BHI). pp. 595-598.

Cimbálník, J., A. Hewitt, G. Worrell and M. Stead. 2018. "The CS algorithm: A novel method for high frequency oscillation detection in EEG." Journal of Neuroscience Methods, vol. 293, pp. 6-16.

Crépon, B., V. Navarro, D. Hasboun, S. Clemenceau, J. Martinerie, M. Baulac, C. Adam, and M. Le Van Quyen. 2009. "Mapping interictal oscillations greater than $200 \mathrm{~Hz}$ recorded with intracranial macroelectrodes in human epilepsy". Brain, vol. 133, pp. 33-45.

Delorme, A. and S. Makeig. 2004. "EEGLAB: an open source toolbox for analysis of single-trial EEG dynamics including independent component analysis". Journal of Neuroscience Methods, vol. 134, pp. 9-21.

Dirodi, M., E. Tamilia, P. E. Grant, J. R. Madsen, S. M. Stufflebeam, P. L. Pearl, and C. Papadelis. 2019. "Noninvasive Localization of High-Frequency Oscillations in Children with Epilepsy: Validation against Intracranial Gold-Standard". In Proceedings of the 201941 st Annual International Conference of the IEEE Engineering in Medicine and Biology Society (EMBC).

Duncan, D., P. Vespa, A. Pitkänen, A. Braimah, N. Lapinlampi, and A. W. Toga. 2019. "Big data sharing and analysis to advance research in post-traumatic epilepsy". Neurobiology of Disease, vol. 123, pp. $127-136$.

Frauscher, B., F. Bartolomei, K. Kobayashi, J. Cimbalnik, M. A. van 't Klooster, S. Rampp, H. Otsubo, Y. Höller, J. Y. Wu, E. Asano, J. Engel, P. Kahane, J. Jacobs, and J. Gotman. 2017. "High-frequency oscillations: The state of clinical research". Epilepsia, vol. 58, pp. 1316-1329.

Garner, R., M. La Rocca, P. Vespa, N. Jones, M. M. Monti, A. W. Toga, and D. Duncan. "Imaging biomarkers of posttraumatic epileptogenesis". Epilepsia, vol. 60, pp. 2151-2162. 
Gliske, S. V., Z. T. Irwin, K. A. Davis, K. Sahaya, C. Chestek, and W. C. Stacey. 2016. "Universal automated high frequency oscillation detector for real-time, long term EEG". Clinical Neurophysiology, vol. 127, pp. 1057-1066.

Gliske, S. V., W. C. Stacey, K. R. Moon, and A. O. Hero. 2016. "The intrinsic value of HFO features as a biomarker of epileptic activity". In Proceedings of the 2016 IEEE International Conference on Acoustics, Speech and Signal Processing (ICASSP).

Jacobs, J., P. LeVan, R. Chander, J. Hall, F. Dubeau, and J. Gotman. 2008. "Interictal high-frequency oscillations $(80-500 \mathrm{~Hz})$ are an indicator of seizure onset areas independent of spikes in the human epileptic brain". Epilepsia, vol. 49, pp. 1893-1907.

Li, L., M. Patel, J. Almajano, J. Engel Jr, and A. Bragin. 2018. "Extrahippocampal high-frequency oscillations during epileptogenesis". Epilepsia, vol. 59, pp. e51-e55.

Liu, S., C. Gurses, Z. Sha, M. M. Quach, A. Sencer, N. Bebek, and N. F. Ince. 2018. "Stereotyped highfrequency oscillations discriminate seizure onset zones and critical functional cortex in focal epilepsy". Brain, vol. 141, pp. 713-730.

Liu, S., N. F. Ince, A. Sabanci, A. Aydoseli, Y. Aras, A. Sencer, and C. Gurses. 2015. "Detection of high frequency oscillations in epilepsy with k-means clustering method". In Proceedings of the 2015 7th International IEEE/EMBS Conference on Neural Engineering (NER).

McHugh M. L. 2012. "Interrater reliability: the kappa statistic". Biochemia medica, vol. 22, pp. 276-282.

Navarrete, M., C. Alvarado-Rojas, M. Le Van Quyen, and M. Valderrama. 2016. "RIPPLELAB: A Comprehensive Application for the Detection, Analysis and Classification of High Frequency Oscillations in Electroencephalographic Signals". PLOS ONE, vol. 11.

Pearce, A., D. Wulsin, J. A. Blanco, A. Krieger, B. Litt, and W. C. Stacey. 2013. "Temporal changes of neocortical high-frequency oscillations in epilepsy". Journal of Neurophysiology, vol. 110, pp. 11671179 .

Smart, O., and M. Chen. 2015. "Semi-automated patient-specific scalp EEG seizure detection with unsupervised machine learning". In Proceedings of the 2015 IEEE Conference on Computational Intelligence in Bioinformatics and Computational Biology (CIBCB).

Spring, A. M., D. J. Pittman, Y. Aghakhani, J. Jirsch, N. Pillay, L. E. Bello-Espinosa, C. Josephson, and P. Federico. 2017. "Interrater reliability of visually evaluated high frequency oscillations". Clinical Neurophysiology, vol. 128, pp. 433-441.

Staba, R. J., C. L. Wilson, A. Bragin, I. Fried, and J. Engel Jr. 2002. "Quantitative Analysis of HighFrequency Oscillations $(80-500 \mathrm{~Hz})$ Recorded in Human Epileptic Hippocampus and Entorhinal Cortex". Journal of Neurophysiology, vol. 88, pp. 1743-1752.

Van Der Maaten, L. 2015. "Accelerating t-SNE using Tree-Based Algorithms". Journal of Machine Learning Research. vol. 15. pp. 3221-3245.

Van Der Maaten, L. and G. Hinton. 2008. "Visualizing Data using t-SNE". Journal Machine Learning Research. vol. 9. pp. 2579-2605.

Vespa, P. M. and M. M. Monti. 2013. "Thalamic atrophy in antero-medial and dorsal nuclei correlates with six-month outcome after severe brain injury". NeuroImage: Clinical, vol. 3, pp. 396-404. 
Worrell, G. A., A. B. Gardner, S. M. Stead, S. Hu, S. Goerss, G. J. Cascino, F. B. Meyer, R. Marsh, and B. Litt. 2008. "High-frequency oscillations in human temporal lobe: simultaneous microwire and clinical macroelectrode recordings". Brain, vol. 131, pp. 928-937.

Worrell, G. A., L. Parish, S. D. Cranstoun, R. Jonas, G. Baltuch, and B. Litt. 2004. "High-frequency oscillations and seizure generation in neocortical epilepsy". Brain, vol. 127, pp. 1496-1506.

Zelmann, R., F. Mari, J. Jacobs, M. Zijlmans, F. Dubeau, J. Gotman. 2012. "A comparison between detectors of high frequency oscillations". Clinical Neurophysiology, vol. 123, pp. 106-116.

Zijlmans, M., P. Jiruska, R. Zelmann, F. S. S. Leijten, J. G. R. Jefferys, and J. Gotman. 2012. "Highfrequency oscillations as a new biomarker in epilepsy". Annals of Neurology, vol. 71, pp. 169-178.

Zuo, R., J. Wei, X. Li, C. Li, C. Zhao, Z. Ren, and X. Zhang. 2019. "Automated Detection of HighFrequency Oscillations in Epilepsy Based on a Convolutional Neural Network". Frontiers in Computational Neuroscience, vol. 7, pp. 82501-82511.

\section{AUTHOR BIOGRAPHIES}

JIAJU LIU is currently a Senior at Palos Verdes Peninsula High School and a researcher at the Laboratory of Neuro Imaging at the USC Stevens Neuroimaging and Informatics Institute. His research interests include using signal processing and unsupervised learning methods to detect and classify high frequency oscillations. His email address is jiajuliu1@gmail.com.

RACHAEL GARNER holds a B.A. in Cognitive Science from the University of California, Berkeley and is currently a Project Assistant at the Laboratory of Neuro Imaging at the USC Stevens Neuroimaging and Informatics Institute. Her research interests include multimodal data analysis related to neurodegenerative disorders, epilepsy, and cognitive disorders. Her email address is rachael.garner@loni.usc.edu.

MARIANNA LA ROCCA received her Ph.D. from Bari University. Currently, she is a Postdoctoral Scholar-Research Associate in the Laboratory of Neuro Imaging at the USC Stevens Neuroimaging and Informatics Institute. Her research interests include image processing, pattern recognition, machine learning techniques, and complex networks applied to neuroscience. Her email address is marianna.1arocca@1oni.usc.edu.

EUN-KEE BAE is an Associate Professor of Neurology at Inha University Hospital and a visiting professor at the Laboratory of Neuro Imaging at the USC Stevens Neuroimaging and Informatics Institute. She holds an M.D and a Ph.D. She has expertise as a clinical epileptologist and specializes in identifying various types of epileptiform activity, including HFOs, in EEG of epilepsy patients. Her email address is eunkee.bae@1oni.usc.edu.

DOMINIQUE DUNCAN is an Assistant Professor of Neurology, Biomedical Engineering, and Neuroscience in the Laboratory of Neuro Imaging at the USC Stevens Neuroimaging and Informatics Institute. She holds a Ph.D. in Electrical Engineering from Yale University. Her research interests include developing analytic tools to study epilepsy, particularly after traumatic brain injury, large-scale multimodal databasing, and combining machine learning and virtual reality for neuroimaging segmentation and analysis. Her email address is dominique.duncan@1oni.usc.edu. 